MS22-O2 Probability densities in different spaces: when multipolar-atom model is just not enough.

Jean-Michel Gillet ${ }^{1}$, Zeyin Yan ${ }^{1}$, Iurii Kibalin ${ }^{2}$, Bolivard Voufack $^{3}$, Nicolas Claiser ${ }^{3}$, Maxime Deutsch ${ }^{3}$, Beatrice Gillon ${ }^{2}$, Claude Lecomte ${ }^{3}$, Pietro Cortona ${ }^{1}$, Alessandro Genoni ${ }^{4}$,

Yoshiharu Sakurai $^{5}$, Masayoshi Itou ${ }^{5},{\text { Masahisa } \text { Itoh }^{6}, \text { Marek }}^{2}$ Brancewicz ${ }^{5}$, Naruki Tsuji ${ }^{5}$, Arsen Gukasov ${ }^{2}$, Florence Porcher ${ }^{2}$, Mohamed Souhassou ${ }^{3}$

1. Laboratoire SPMS, UMR CNRS 8580, CentraleSupelec, Université Paris-Saclay, France

2. Laboratoire Léon Brillouin CEA/CNRS, France

3. Laboratoire CRM2, UMR CNRS 7036, Université de Lorraine, France

4. Laboratoire SRSMC, CNRS \& Université de Lorraine, France

5. JASRI/ SPring8, Japan

6. Gunma University, Japan

email: Jean-michel.gillet@centralesupelec.fr

In many situations, multipolar-atom refinement model is still the most efficient means of reconstructing charge density in position space from high resolution X-ray diffraction (XRD) data. Furthermore, polarized neutron diffraction (PND), which is a unique technique for the study of unpaired electron in magnetic systems, has also beneficiated from the great versatility of the multipole on pseudo-atom model. Providing that the description on "magnetic atoms" allows for a differentiation depending on the spin state, we have demonstrated that a joint analysis of polarized neutron and X-ray diffraction data is also possible. A wealth of other experimental data has been made available by a development of more powerful sources. For example, magnetic X-ray diffraction (MXRD) or magnetic Compton scattering (MCS) are such techniques that have developed almost independently with the advent of efficient insertion devices in synchrotron facilities. Because MCS relies on an incoherent inelastic process it is better adapted to a representation in momentum space. Such a technique is thus a valuable means for observing the most delocalized unpaired electrons. There have been numerous demonstrations that a mere incoherent addition of atom centred contributions is not well adapted to momentum space description. As a consequence, the ambition to gather data from XRD, PND, MXRD and MCS constitutes a new motivation to explore beyond multipole on pseudo-atom models. The talk will address this new and exciting challenge through our latest results concerning spin and charge densities in YTiO3.

Financial support from ANR (MTMED project) and CNRS are gratefully acknowledged.

Ref: "First spin-resolved electron distributions in crystals from combined polarized neutron and X-ray diffraction experiments" IUCrJ (2014), Volume 1, Part 3, Pages 194-199, M. Deutsch, B. Gillon, N. Claiser, J.-M. Gillet, C. Lecomte and M. Souhassou,

Keywords: X-ray diffraction, polarized neutron diffraction, magnetic Compton scattering, ab-initio computation, charge density, spin density, position space, momentum space, ferromagnet

\section{MS22-03 Electron Density of a Layered Transition Metal Dichalcogenide}

Hidetaka Kasai ${ }^{1,2}$, Kasper Tolborg ${ }^{2}$, Mattia Sist ${ }^{2}$, Venkatesha R. Hathwar $^{2}$, Mette O. Filsoe ${ }^{2}$, Simone Cenedese ${ }^{2}$, Kunihisa Sugimoto $^{3}$, Jacob Overgaard ${ }^{2}$, Eiji Nishibori ${ }^{1}$, Bo B. Iversen ${ }^{2}$

1. Faculty of Pure and Applied Sciences, TIMS and CiRfSE, University of Tsukuba

2. Center for Materials Crystallography, Department of Chemistry and iNANO, Aarhus University

3. Japan Synchrotron Radiation Research Institute

email: kasai.hidetaka@chem.au.dk

Electron density (ED) studies enable us to understand chemical bonding features in materials. While accurate experimental ED has provided information on bonding in a variety of materials, it is still a great challenge to evaluate weak interactions e.g. in layered structures. Layered transition metal dichalcogenides (TMDs) have attracted much attention because of their unique properties e.g. as topological insulators [1], charge density wave systems [2], electrode materials for ion batteries [3], and thermoelectric materials [4]. The generalized formula is $\mathrm{MX}_{2}$, where $\mathrm{M}$ is a transition metal and $\mathrm{X}$ is a chalcogen ( $\mathrm{S}, \mathrm{Se}$ and $\mathrm{Te}$ ). The layered structure exhibits strong covalent intralayer bonding and weak van der Waals (vdW) interaction between adjacent layers. The weak vdW interaction also enables stacking different TMDs layers [5] as well as application of a monolayer or a few-layer TMDs [6]. Understanding the weak interaction in TMDs is important not only for fundamental science but also for application. Recently the interlayer interaction in $\mathrm{SnS}$, was discussed using high pressure techniques [7]. In the present study, we evaluate the weak interlayer interaction in TMDs using experimental X-ray ED. We measured single crystal diffraction data of TiS (Space Group: $P-3 m 1$ ) to a resolution of $\sin \theta / \lambda=1^{2} .67 \AA^{-1}$ at $20 \mathrm{~K}$ using $50 \mathrm{keV}$ $\mathrm{X}$-ray with the image plate detector at BL02B1 of SPring-8. The ED was modelled with the extended Hansen-Coppens multipole model [8], which includes refinement of core electron density features. In the presentation, we will discuss the weak interlayer interaction based on the ED.

[1] X. Qian et al., Science 346, 1344 (2014).

[2] J. Okamoto et al., Phys. Rev. Lett. 114, 026802 (2015).

[3] A. Unemoto et al., Chem. Mater. 27, 5407 (2015).

[4] C. Wan et al., Nature Mater. 14, 622 (2015).

[5] A. K. Geim and I. V. Grigorieva, Nature 499, 419 (2013).

[6] M. Chhowalla et al., Nature Chem. 5, 263 (2013).

[7] M. Ø. Filsø et al., Dalton Trans. 45, 3798 (2016).

[8] A. Fischer et al., J. Phys. Chem. A 115, 13061 (2011).

Keywords: Electron density, layered structure, multipole model 